\title{
On the Distinction between Debye Bosons and Acoustic Phonons
}

\author{
U. Köbler \\ Research Centre Jülich, Institute PGI, 52425 Jülich, Germany \\ E-mail: u.koebler@fz-juelich.de
}

Received 20 July 2015, Revised 25 November 2015, Accepted 28 November 2015

\begin{abstract}
A method is described that allows one to construct the dispersion of the Debye bosons (sound waves) from the known temperature dependence of the sound velocities. The method simply assumes that the sound velocity measured at temperature $T$ gives the slope of the dispersion relation at excitation energy $E=k_{B} T$. The associated reduced wave vector is set equal to $q / q_{0}=a_{0} k_{B} T / h v_{L} / T$. In this way the dispersion of the Debye bosons can be constructed for all thermal energies for which sound velocities $v_{L} / T(T)$ are known. This can be up to melting temperature. Surprisingly, for metals and for insulators the dispersion of the Debye bosons can continue up to wave vector values of several times the zone boundary. At melting temperature the wavelength of the Debye bosons is of the order of the atomic diameters. The sources of the Debye bosons therefore must have atomic dimensions. Spontaneous generation of Debye bosons by individual atoms is, however, a completely unexplored process. Interactions with the atomistic background of phonons or lattice defects provide damping to the Debye bosons and make the material specific sound velocity $v_{L} / T(T)$ additionally sample and temperature dependent. For practically all solids it is observed that elastic constants and $v_{L} / T(T)$ decrease as a function of increasing temperature. For high energies the dispersion relation of the Debye bosons therefore becomes visibly lower than linear. Interactions between Debye bosons and phonons can modify the dispersion of the acoustic phonons appreciably. Because of their different symmetries the dispersion relations of Debye bosons and acoustic phonons can attract each other. It is observed that for low wave vector values the dispersion of the acoustic phonons can assume the linear wave vector dependence of the Debye bosons. At the end of the linear section a functional crossover to a sine-like function of wave vector occurs.
\end{abstract}

Keywords: Sound velocity; acoustic phonons; boson fields.

\section{Introduction}

Sound propagation in solids, liquids and gases belongs to those dynamic phenomena in which atoms and interactions between atoms are not involved (directly). The identical propagation mode in all three phases of matter illustrates that sound waves are not coupled to the thermal motion of the atoms. Independence of atomistic degrees of freedom commonly is called universal. Universality is well known from the dynamics in the vicinity of critical temperatures. Instead of atomistic parameters (lattice constant etc.) the material specific mass density seems to be the only important parameter for sound propagation. Evidently, sound waves are distinguished energy degrees of freedom that are specific to the continuous elastic medium. Note that there are no atoms to be considered in the continuous elastic medium. In this communication we will call the sound waves in crystalline solids Debye bosons. Since Debye bosons are mass less particles they cannot be observed using inelastic neutron scattering. This makes experimental determination of the dispersion relation of the Debye bosons difficult. Here we propose a method to obtain the dispersion relation of the Debye bosons for all energies and wave vector values. The method simply assumes that the sound velocity measured at temperature $T$ gives the slope of the dispersion relation at thermal energy of $k_{B} T$. In this way it is possible to construct the dispersion of the Debye bosons for all thermal energies for which sound velocities are known. This can be up to solid-liquid transition at melting temperature $T_{m}$. Note that melting temperature commonly is much larger than conforms to the largest phonon energy. The largest phonon energy can be characterized by $k_{B} \Theta \mathrm{D} \quad(\Theta \mathrm{D}=\mathrm{Debye}$ temperature). Since $T_{m}>\Theta D$ the dispersion of the Debye bosons can reach energies of several times larger than phonon energy. Moreover, the dispersion of the Debye bosons continues much beyond zone boundary. This is because lattice parameter and zone boundary are of no importance for the ballistic propagation mode of sound waves.

That sound waves and phonons are independent energy degrees of freedom was an implicit assumption of Debye's field theory of the low temperature heat capacity of solids [1]. In his theory of 1912 P. Debye made, tacitly, two not self-evident assumptions: first, that the dynamics of massive interacting atoms can be replaced by the dynamics of noninteracting mass-less bosons (the ultrasonic waves) and, second, that all thermal energy is not in the system of the interacting atoms but is in the field of freely propagating bosons. It then suffices to consider the energy degrees of freedom of the field exclusively. The heat capacity of the solid is the heat capacity of the boson field $\left(\sim T^{3}\right)$. Under this condition atomistic structures are unimportant. This is the origin of universality. In fact, universality (in the sense of field theory) is the thermodynamic behavior of a field of freely propagating bosons. As a consequence, one has to distinguish between the thermal reservoir of the boson field and the thermal reservoir of the atomistic lattice. Coupling between the two systems can be assumed to be weak. As a further consequence, the two systems must have different 
excitation spectra with different dispersion relations. As we now know, the boson field is the relevant thermal reservoir only below some crossover temperature at which thermal energy changes from phonon system to boson field [2]. This crossover commonly is at a temperature of $10 \ldots 20 \mathrm{~K}$.

Crossover events were unknown at the time of Debye. This was essentially because the necessity to distinguish between the discrete translation symmetry of the atomistic solid and the continuous translation symmetry of the continuous solid was not yet recognized. This has contributed to a long standing misinterpretation of Debye's field theory. Even today Debye's field theory is widely considered as a low temperature approximation of lattice theory. As we will see this view is not correct by symmetry arguments. We have to attribute different translational symmetries (discrete and continuous) to phonons and Debye bosons. The different symmetries exclude that the two thermal reservoirs come simultaneously into play. At crossover of thermal energy to phonon system field theory has to be replaced by lattice theory. Atomistic lattice theories then are appropriate [3]. We should note that the Debye boson field has much similar with the electromagnetic radiation field in that the dispersion relations of the associated field particles are linear functions of wave vector and the energy densities of the two fields are $\sim T^{4}$.

The two assumptions made unwittingly by Debye in 1912 received an indirect justification only in 1974 through development of Renormalization Group (RG) theory mainly by K.G. Wilson [4,5]. As was shown by RG theory, in ordered magnets the dynamics of interacting (massive) spins has to be replaced by the dynamics of a boson field. One of the main issues of RG theory was that on approaching the magnetic ordering temperature from the paramagnetic side, spins and interactions between spins become unimportant for the dynamics (of the spins!). In other words, spin dynamics in ordered magnets is another example of a thermodynamic phenomenon that is independent of atomistic structures. Somewhat above ordering temperature thermal energy passes via a crossover event from the system of the interacting spins (in the Curie-Weiss regime of susceptibility) to a field of freely propagating bosons (in the critical paramagnetic range and below). The dynamics of the spins then is the dynamics of a boson field and therefore shows universality [6]. Due to a weak coupling between field and spins the nonrelevant spins receive their dynamics from the boson guiding field. This allows one to study the dynamics of the field by observation of the spin degrees of freedom. In fact, thermal decrease of the magnetic order parameter is controlled by the heat capacity of the boson field and not by inter-atomic exchange interactions. The nonrelevant spin system does virtually not contribute to the magnetic heat capacity. The heat capacity of the magnetic system is the heat capacity of the boson field [2,6]. RG theory could, however, not clarify the nature of the field bosons in ordered magnets. Note that the Debye boson field is the only boson field of solid state physics for which the field quanta (sound waves) are well known from practical experience. As we have argued elsewhere [6], the field quanta controlling the spin dynamics in ordered magnets are essentially magnetic dipole radiation emitted upon precession of the magnetic moments. The sources of the field quanta are the atomic magnetic moments, the field has radiation character. Not only in ordered magnets but also in conventional superconductors the dynamics seems to be determined by boson fields $[2,6]$.
In diamagnetic solids the functionality of the magnetic ordering temperature has shifted to $T=0$. Only in the "critical" range above $T=0$ (for temperatures below $10 \ldots 20$ $\mathrm{K})$ the heat capacity of the solid is the heat capacity of the Debye boson field and the heat capacity follows $T^{3}$ function.

Quite generally, independence of the dynamics of atomistic degrees of freedom (universality) indicates that the dynamics is that of a boson field. Freely propagating bosons have dispersion relations that are a simple power function of wave vector for all energies. This results into a finite temperature range for the universal power function of absolute temperature for the heat capacity of the boson field. In fact, boson dynamics is easily recognized by the finite validity range of the critical power functions. Note that the power functions predicted by atomistic theories such as Bloch's $T^{3 / 2}$ function for the thermal decrease of the spontaneous magnetization of the isotropic ferromagnet hold asymptotically at $T=0$ only. Non universality of the $T^{3 / 2}$ function reveals further by the fact that it applies to ferromagnets but not to antiferromagnets. Experimentally it is observed that thermal decrease of the magnetic order parameter is universal, that is, independent of spin structure $[2,6]$. It is evident that the asymptotic exponents calculated by atomistic models must generally be different from the observed universal exponents of the field dynamics. Atomistic models such as the Brillouin function give only a very crude account of the spontaneous magnetization.

Debye bosons and phonons are distinguished by different propagation modes. Note that the propagation mode defines the dispersion relation of the particles. While field quanta propagate ballistic, independent of atomistic structures, phonons propagate from lattice site to lattice site. In other words, field bosons and phonons are distinguished by different translational symmetries (continuous and discrete). The two translational symmetries are, so to say, the generators of the two particles. What we have to learn is that continuous translational invariance of the infinite solid has to be accepted as a specific, particle generating symmetry. This symmetry applies to all crystalline solids and entails excitation spectra in addition to the atomistic excitations. Phonons are the excitations of the discrete atomistic lattice while Debye bosons are the excitations of the elastic continuum. Continuous translational invariance of the elastic continuum implies that the momenta of the Debye bosons are conserved quantities.

Normally bosons become the relevant excitations only in the vicinity of a critical temperature. Note in particular that $T=0$ is also a critical point with boson dynamics over a finite range of absolute temperature. It is important to note that the different translational symmetries of Debye bosons and phonons apply to all wave vector values. Rotational degrees of freedom commonly are local modes and do not become relevant for the universal (long range) boson dynamics. In other words, by symmetry reasons field theory and lattice theory never become identical even in the limit of an infinite wavelength. This does not exclude that, by chance, the critical exponents predicted by the two theories can be identical. However, the critical power functions of the atomistic theories hold asymptotically only. As a consequence of their different symmetries, the dispersion relations of the two particles are well distinguished for all energies even when their dispersions become identical for small wave vector values. Note that inelastic neutron scattering is sensitive to phonons only. This is because impact of neutrons is a microscopic excitation process by 
which local atomistic modes are excited. Sound injection into the solid evidently is a macroscopic excitation process whereby bosons are generated.

Since boson field and lattice system have different excitation spectra we may ask: which excitation spectrum defines the experimentally observed heat capacity. At this point another important issue of RG theory becomes important: the principle of relevance. Due to their different symmetries the two excitation spectra become relevant alternatively only. Relevance therefore can be considered as a dynamic symmetry selection rule. In other words, thermal energy is either in the boson field or in the atomistic system of phonons. Relevance has the dramatic consequence that the population of all available energy states of field and atomistic system cannot be according to the Boltzmann factor but must be subject to a selection rule. When phonons are the relevant excitations the boson field accumulates no longer thermal energy and its heat capacity tends to zero. The fact that the heat capacity of all solids reasonably saturates at atomistic Dulong-Petit limit shows that the $T^{3}$ function of the heat capacity of the Debye boson field has dropped to zero. In other words, at ambient temperature the dispersion relation of the Debye bosons is no longer thermally populated and its heat capacity is negligible. The important consequence of relevance is that at any temperature the dynamics can be classified by one or the other symmetry. A mix of symmetries does not occur. Which system is relevant depends on which system has the lower excitation energy. Since the dispersion of the Debye bosons essentially remains linear for all energies but the dispersion of the acoustic phonons saturates towards zone boundary, phonons have lower excitation energies at ambient temperature and are the relevant excitations. Only for temperatures of lower than $10 \ldots 20 \mathrm{~K}$ Debye bosons are relevant, and the heat capacity of phonons is negligible. We can assume that the heat capacity of the boson field essentially drops to zero at crossover of thermal energy to phonons at $10 \ldots 20 \mathrm{~K}$. On the other hand, the energy states of the phonon system become depopulated below crossover to field dynamics.

The two dynamic symmetries do, however, not hold perfectly. As is well known, symmetry violations are quite common in all parts of physics. Nevertheless, symmetries are indispensable basic concepts to start with. Debye boson field and phonon system are not independent of each other but interact visibly. Interactions of Debye bosons either with phonons or lattice defects provide damping to the Debye bosons and have two effects: they shorten the mean free path of the Debye bosons and decrease their velocity. In fact, decrease of sound velocity with increasing temperature is a clear indication of phonon-Debye boson interaction and/or scattering at lattice defects [7]. As a consequence, the dispersion of the Debye bosons increases slightly lower than linear. If Debye bosons would propagate unperturbed their mean free path would be infinite and sound velocity would be a constant for all temperatures. In this case the dispersion would be exactly linear for all energies. This is as for the electromagnetic radiation field in vacuum. One could call the Stefan-Boltzmann T4 function for the energy density of the electromagnetic radiation field universal because it is due to a boson field (photons) and holds over a large temperature range. Only for extremely high photon energies deviations from linearity could become visible.

Another clear indication of interactions between Debye bosons and phonons is given when the dispersion of the acoustic phonons starts not as sine function of wave vector but as a linear function of wave vector over a considerable wave vector range. Because the slope of the linear section agrees with the measured sound velocity it can be concluded that phonon dispersion has adapted to the linear dispersion of the Debye bosons. Note that dispersion lines of particles with different symmetries (discrete and continuous) can attract each other. Dispersion lines of particles with the same symmetry avoid each other. Interestingly, boson dispersion attracts phonon dispersion and not vice versa. Linearity in the acoustic phonon dispersion can hold up to excitation energies that are much larger than corresponds to the validity range of the $T^{3}$ function of the heat capacity of the boson field. This allows verification of the linear dispersion of the Debye bosons from the known phonon dispersions up to energies where Debye bosons are no longer relevant, and the heat capacity of the boson field is zero. This shows that phonon dispersion can be attracted by the thermally unoccupied boson dispersion.

At the end of the linear section of the phonon dispersion an analytical crossover to sine function of wave vector follows. However, in order to get good phenomenological description of the experimental data it is necessary to add a phase shift to the argument of the sine function. The phase shift indicates finite Debye boson-phonon interactions for all q-values. In order to understand the phase shift we have to consider that for freely propagating bosons lattice parameter and zone boundary have no significance. Insignificance of zone boundary for the boson field gets transferred partly to the phonon system via interaction of the two systems and can rationalize the phase shift. The phase shift can be considered as a measure of the strength of Debye boson-phonon interaction. It is evident that for strong Debye boson-phonon interaction lattice theories cannot give correct description of the phonon dispersions. In the dispersion relation of the Debye bosons the corresponding functional crossover events are much weaker than in the phonon dispersions (see Figure 3 and Figure 5).

\section{Analysis of Experimental Data}

Debye boson-phonon interactions occasionally are weak and do not produce anomalies in the phonon dispersions. Under this condition lattice theories can give reasonable description of the phonon dispersions [3]. The rare gas solids [8-11] and $\mathrm{CsCl}$ [12] are examples of a vanishing Debye boson-phonon interaction. For these solids the acoustic phonons are perfectly described by sine functions of wave vector. This conforms to lattice theory for inter-atomic forces limited to nearest neighbors.

In most solids Debye boson-phonon interaction is stronger for longitudinal polarization than for transverse polarization. Often the acoustic phonons with transverse polarization follow perfect sine function of wave vector. As an example Figure 1 shows neutron scattering data of the acoustic phonons for $\mathrm{LiF}$ measured along cube edge at room temperature [13]. For transverse polarization (dots) phonon dispersion is excellently described by sine function of wave vector (solid curve). Interactions with Debye bosons therefore are weak. Consistent with weak interactions between Debye bosons and phonons is a relatively weak temperature dependence of sound velocity $v_{\mathrm{T}}(T)$ (compare Figure 3 and Figure 5). The initial slope of the sine function agrees perfectly with the measured sound velocity [14]. Note that sound velocity and phonon dispersions are measured by completely different experimental methods on different samples. 


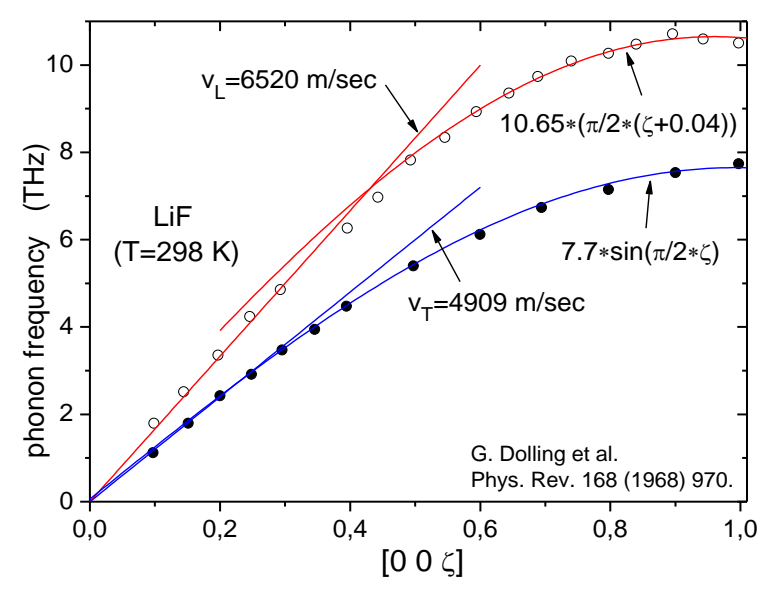

Figure 1. Dispersions of the acoustic phonons with transverse $(T)$ and longitudinal $(L)$ polarization of LiF measured along cube axis at T=298 K [13]. For transverse polarization (dots) phonon dispersion is perfectly described by sine function of wave vector. In the dispersion relation with longitudinal polarization (circles) a functional crossover from linear dependence to sine-like dependence occurs. This is indicative of Debye boson-phonon interaction (see text).

For longitudinal polarization (circles), phonon dispersion deviates visibly from sine function. In conformity with many empirical analyses of phonon dispersion data, the phonon branch with longitudinal polarization consists of two sections with different functions of wave vector. This behavior can be called an analytical or functional crossover. An analytical crossover is indicative of two contributions to the phonon dispersions. In fact, in the low wave vector range a fairly linear function of wave vector can be seen. The slope of this line agrees reasonably with the measured sound velocity [14]. Note that sound velocities (and elastic constants) depend somewhat on the crystal perfection of the investigated sample. Beyond analytical crossover at about $0.43 \mathrm{q} / \mathrm{q}_{0}$ phonon dispersion is well described by sine function of wave vector. However, adequate description of experimental data requests that a phenomenological phase shift is added to the argument of the sine function. The phase shift is a general phenomenon in materials with strong Debye boson-phonon interaction (see also Figure 7). For LiF the phase shift amounts to $+4 \%$ only and is relatively small compared to the examples to follow (compare Figure 6). We therefore defer discussion of this point to the following examples with a much larger phase shift. Evidently, the phase shift is a measure for the Debye boson-phonon interaction strength. In other words, a strong Debye boson phonon interaction is noticeable for all wave vector values.

As a conclusion, the phonon dispersion curve with longitudinal polarization consists of two sections. In the low wave vector range the phonon dispersion is defined by Debye bosons and in the large wave vector range by interatomic forces. It is evidently not possible to describe the whole phonon dispersion branch by only one theory. Similar analytical crossover events can be identified in the magnon dispersions of magnetic materials [15].

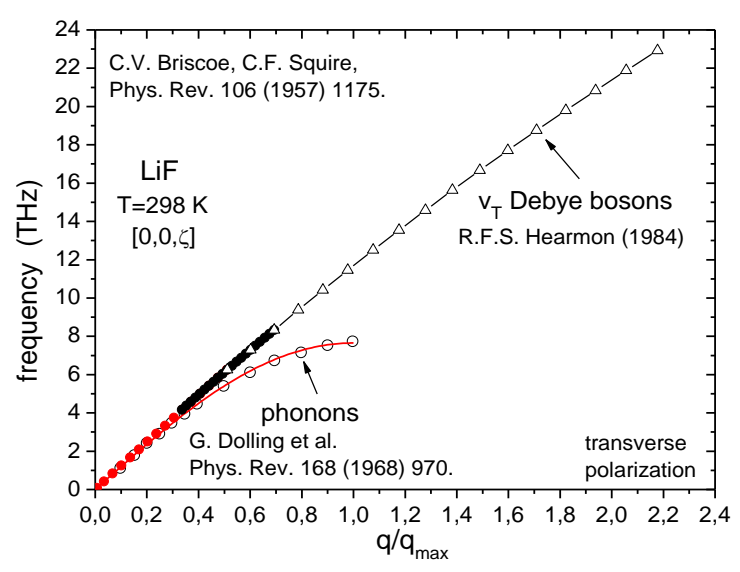

Figure 2. Dispersion of the acoustic phonons with transverse polarization (circles) of LiF measured along cube edge at $T=298$ K [13]. For transverse polarization Debye boson-phonon interaction is weak. Sound velocity $v_{T}$ is only weakly temperature dependent and Debye boson dispersion extends nearly linearly up to $q / q_{\max }=2.18$. Construction of the dispersion of the Debye bosons from sound velocity data up to melting temperature $\left(T_{m}\right)$ is given by filled symbols and by triangles $[7,14]$. Note that $T_{m}=1121 \mathrm{~K}$ corresponds to $\approx 23.3 \mathrm{THz}$. At $T_{m}$ the wavelength of the Debye bosons is 1.84 $\AA\left(a_{0}=4.0173 \AA\right)$.

Figure 2 extends the dispersion of the Debye bosons with transverse polarization to larger wave vector values (and energies) than Figure 1. Note that the abscissa expands up to $\sim 2.18$ times the zone boundary. Figure 2 compiles calculated dispersion data of the Debye bosons with transversal polarization from different sound velocity data sets (dots [14], triangles [7]).

As we have mentioned, for the construction of the dispersion of the Debye bosons from the known sound velocities we set the dispersion energy of the Debye bosons equal to $E=k_{B} T$ with $T$ as temperature at which sound velocity has been measured. Sound velocities $v_{\mathrm{L} / \mathrm{T}}(\mathrm{T})$ therefore enter the expression of the reduced wave vector $\mathrm{q} / \mathrm{q}_{0}$ only. Considering that at zone boundary the energy of the sound waves is $E=v_{\mathrm{L} / \mathrm{T}} \cdot h / a_{0}$ with $h=$ Planck's constant and $a_{0}=$ lattice parameter, the complete expression for the dispersion relation of the sound waves reads $E=k_{B} T$ $=v_{\mathrm{L} / \mathrm{T}} \cdot h / a_{0} \cdot q / q_{0}$. As reduced wave vector one obtains: $q / q_{0}=$ $a_{0} \cdot k_{B} T / h \mathrm{v}_{\mathrm{L} / \mathrm{T}}$.

It is evident that for constant sound velocity the dispersion energy of the Debye bosons $\left(k_{B} T\right)$ is proportional to the reduced wave vector $q / q_{0}$. The dispersion is exactly linear. For decreasing sound velocity as a function of increasing temperature the reduced wave vector increases stronger than dispersion energy $k_{B} T$. The dispersion of the Debye bosons therefore becomes slightly weaker than linear. Figure 2 includes the calculated dispersion of the Debye bosons using the known data for the temperature dependence of the sound velocity $v_{T}(T)[7,14]$.

As can be seen in Figure 2, the dispersion of the Debye bosons reaches energies of much larger than phonon energy and continues up to 2.18 times of zone boundary. Note that $T_{m}=1121 \mathrm{~K}$ but $\Theta_{\mathrm{D}}=740 \mathrm{~K}$. At zone boundary $\left(q / q_{\max }=1\right)$ no definite anomaly is visible in the dispersion of the Debye bosons. This conforms to the lattice structure independent 
propagation mode of sound waves. Note that for energies of the zone boundary the dispersion of the Debye bosons is no longer thermally populated. Beyond zone boundary sound waves have wavelengths of shorter than lattice parameter. Dispersion until $q / q_{\max }=2.18$ indicates that the sources of the Debye bosons must have dimensions of the order of the atomic diameters. From $q / q_{0}=2.18$ at dispersion energy of $k_{B} T_{m}\left(T_{m}=1121 \mathrm{~K}\right)$ it follows that at $T_{m}$ the wavelength of the Debye bosons is $\lambda_{\mathrm{T}}\left(T_{m}\right)=1.84 \AA$ using $a_{0}=4.0173 \AA$ as lattice parameter. The calculated value for $\lambda_{T}$ conforms to the atomic diameters $[16,17]$. Since Debye's low temperature $T^{3}$ function is observed also in strongly anisotropic solids the Debye boson field is an isotropic (scalar) field, and the sources of the field quanta must be essentially spherical. It is therefore suggestive to identify the nearly spherical atomic cores as the sources of the Debye bosons $[16,17]$. On the other hand, nothing is known about the elastic properties of single atoms. It is only clear that gravitational forces are essential in the atomic shell. In particular, it is completely unexplored how atoms can emit sound waves. This problem has to be solved by a future quantum elastodynamics in analogy to the well-developed quantum electrodynamics. It is, however, clear that all elastic properties of solids are macroscopic phenomena that have to be ascribed to Debye bosons and not to the discrete atomistic lattice. This becomes evident from the simple relation between elastic constants and sound velocities. Only the anisotropy of the elastic continuum has atomistic origin.

According to the weak temperature dependence of $v_{T}(T)$ (Figure 3) the dispersion of the Debye bosons deviates fairly weakly from linearity. In fact, $v_{T}(T)$ is perfectly temperature independent up to a rather sharp crossover temperature at $T_{C O}$ $=195 \mathrm{~K}$. At this thermal energy $(\sim 4 \mathrm{THz})$ the dispersions of Debye bosons and phonons start deviating visibly from each other (see Figure 2). Unfortunately phonon dispersions are not accurate enough to reveal a similarly weak analytical crossover as in Figure 3. Instead, fit by sine function holds with good accuracy for all wave vector values.

The stronger Debye boson-phonon interaction for longitudinal polarization manifests in a twofold way: as we have seen in Figure 1, the acoustic phonons do no longer follow perfect sine function of wave vector and the velocity of the sound waves shows stronger temperature dependence than for transverse polarization (compare Figures 3 and 5). As a consequence, the dispersion of the Debye bosons is stronger curved (compare Figures 2 and 4). Comparison of Figures 2 and 4 shows that for longitudinal polarization the phonon dispersion is visibly attracted by the Debye boson dispersion. It is evident that due to the functional crossover phonon dispersion relation cannot be described by lattice theory alone. As will be shown in Figure 8, for a very strong Debye boson-phonon interaction phonons can assume the linear dispersion of the Debye bosons up to zone boundary.

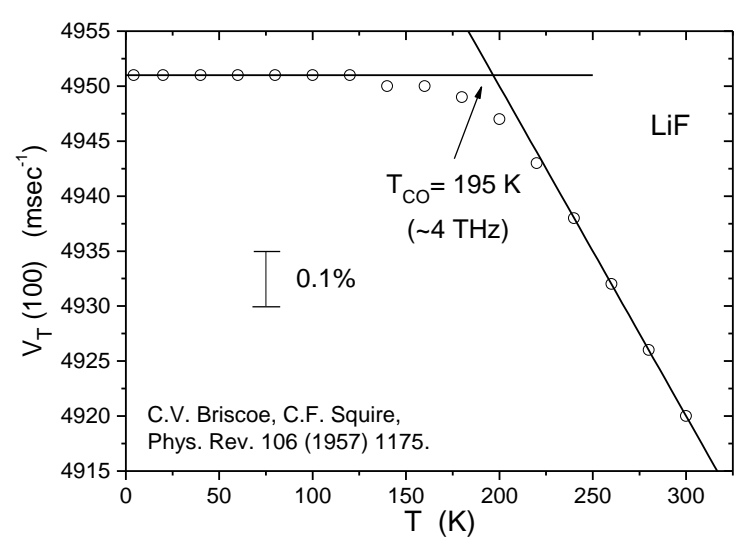

Figure 3. Temperature dependence of sound velocity for sound waves with transverse polarization of LiF [14]. In the temperature range up to crossover temperature $T_{C O}=195 \mathrm{~K}$ $v_{T}$ virtually is temperature independent, and the dispersion of the Debye bosons is exactly linear. For $T>195 \mathrm{~K}$ sound velocity decreases nearly linearly with temperature. This gives rise to a weak quadratic bending of the dispersion of the Debye bosons. Note the ten times stronger temperature dependence for longitudinal polarization (Figure. 5).

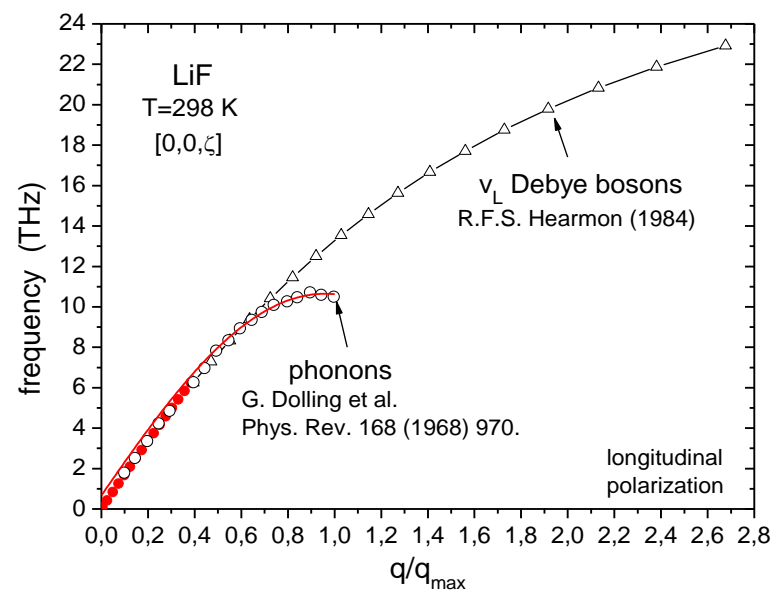

Figure 4. Dispersion of the acoustic phonons with longitudinal polarization measured at room temperature along cube edge of LiF (circles) [13]. For longitudinal polarization Debye boson-phonon interaction is stronger than for transverse polarization. This gives rise to a stronger curvature of the calculated dispersion of the Debye bosons (triangles). Phonon dispersion is visibly attracted by the Debye boson dispersion. Boson dispersion reaches wave vector values of up to $q / q_{\max }=2.7$. 


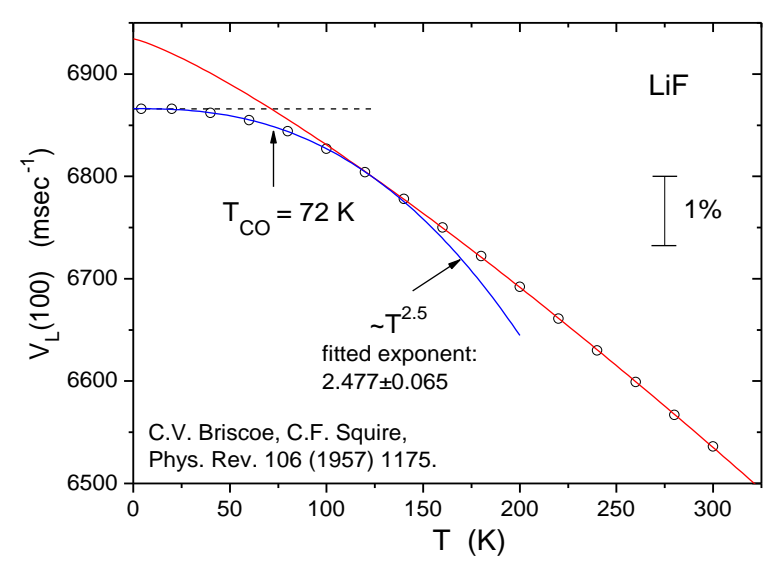

Figure 5. Velocity of sound waves with longitudinal polarization traveling along cube edge of LiF as a function of temperature [14]. The low temperature portion can be fitted by $\sim T^{2.5}$ function. There is no finite temperature range with exactly constant sound velocity. Note the stronger temperature dependence than for transverse polarization in Figure 3.

Quantitative interpretation of the temperature dependence of the sound velocities is difficult because we have to distinguish between an intrinsic scattering of sound waves due to interaction with phonons and a sample dependent scattering due to interaction with impurities and lattice defects. Impurity scattering makes experimental data of the sound velocities (and of the elastic constants) badly reproducible [7]. Nevertheless crossover events between different functions of temperature can often be identified in the temperature dependence of the sound velocities. In Figure 5 crossover from low temperature $T^{2.5}$ function to linear function can be identified. As a consequence, sound velocity is not really temperature independent even for $T \rightarrow 0$. This lets the dispersion of the Debye bosons deviate weakly from linearity just from the beginning and entails weak deviations from $T^{3}$ function in the heat capacity of the Debye boson field.

At melting temperature of LiF the velocity of the Debye bosons with longitudinal polarization is $v_{L}=3328 \mathrm{~m} / \mathrm{sec}$. The wavelength of the Debye bosons therefore is $\lambda_{L}\left(T_{m}\right)=1.42$ A.

Very interesting phonon dispersions are observed for niobium [18]. Niobium is an exception in that Debye bosonphonon interaction is stronger for transverse polarization. Figure 6 shows neutron scattering data along face diagonal of $\mathrm{Nb}$. In the acoustic phonon branch with transverse polarization (crosses) the analytical crossover from linear dispersion to sine function is unusually pronounced. The fitted phase shift in the argument of the sine function is as large as $-20 \%$ of the wave vector value at zone boundary. For the branch with longitudinal polarization the corresponding phase shift is $+7.5 \%$. Dispersion of the Debye bosons calculated from the temperature dependence of the sound velocities is given by dots [19].

As can be seen in Figure 6, the energy of the Debye bosons with transverse polarization is $\sim 3.9 \mathrm{THz}$ at zone boundary. This corresponds to a temperature of $\sim 190 \mathrm{~K}$. In other words, for the construction of the dispersion of the Debye bosons with transverse polarization sound velocity data of the range $0<T<190 \mathrm{~K}$ were needed only. Sound velocities have, of course, been measured up to much higher temperatures [7] (melting temperature of $\mathrm{Nb}$ is $T_{m}=2741 \mathrm{~K}$, $\sim 57 \mathrm{THz}$ ). As for LiF the dispersion of the Debye bosons for $\mathrm{Nb}$ continues much beyond zone boundary. At $T_{m}$ the velocity of sound waves with transverse polarization is $\mathrm{v}_{\mathrm{T}}\left(T_{m}\right)=1933 \mathrm{~m} / \mathrm{sec}$. The wavelength is $\lambda_{T}=.35 \AA$. From $v_{L}\left(T_{m}\right)=4736 \mathrm{~m} / \mathrm{sec}$ it follows $\lambda_{L}\left(T_{m}\right)=0.82 \AA$. The lattice parameter is $\mathrm{a}_{0}=3.30 \AA$. As a consequence, for metallic $\mathrm{Nb}$ with high melting temperature the wavelengths of the Debye bosons at melting temperature are distinctly shorter than for insulating LiF.

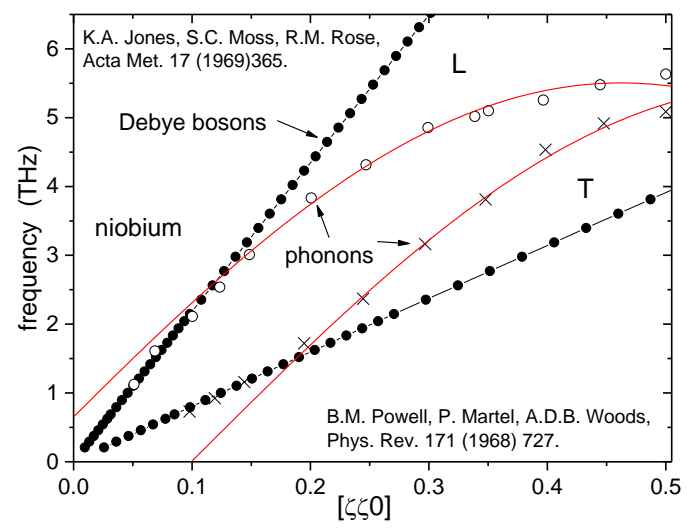

Figure 6. Acoustic phonon dispersions of niobium measured along face diagonal at $T=300 \mathrm{~K}$ (crosses, open points) [18]. In the branch with transverse polarization $(T)$ the analytical crossover from linear dispersion to sine function (including a phase shift) is unusually pronounced. Dispersion of the Debye bosons calculated from sound velocities are given by dots [19]. Since in the energy range shown of up to $6.5 \mathrm{THz}$ $(\sim 312 \mathrm{~K})$ sound velocities depend only weakly on temperature, deviations from linear dispersion can nearly not be recognized.

$\mathrm{NaI}$ is another example of unusually strong Debye bosonphonon interactions [20]. As for most solids Debye bosonphonon interaction is strongest for longitudinal polarization. The linear section in the dispersion of the acoustic phonon branch with longitudinal polarization holds up to an enormous large energy of $\sim 2 \cdot \mathrm{THz}$. This corresponds to a thermal energy of $96 \mathrm{~K}$. At this thermal energy phonons are the relevant excitations and the Debye boson dispersion is no longer thermally populated. For transverse polarization the linear section of dispersion holds up to much smaller energies only. Beyond linear section description by sine function of wave vector including a phase shift gives reasonable description of the experimental data. The fitted phase shift is another measure for the strength of Debye boson-phonon interaction. As a conclusion, Debye bosonphonon interaction opens the unique opportunity to verify the linear dispersion of the Debye bosons using inelastic neutron scattering. The possibility of exciting sound waves at all temperatures, out of thermal equilibrium, shows that the nearly linear dispersion of the Debye bosons must continue beyond the linear section in the phonon dispersion. As a conclusion, interaction of phonons with an empty Debye boson dispersion, therefore, proves possible. 


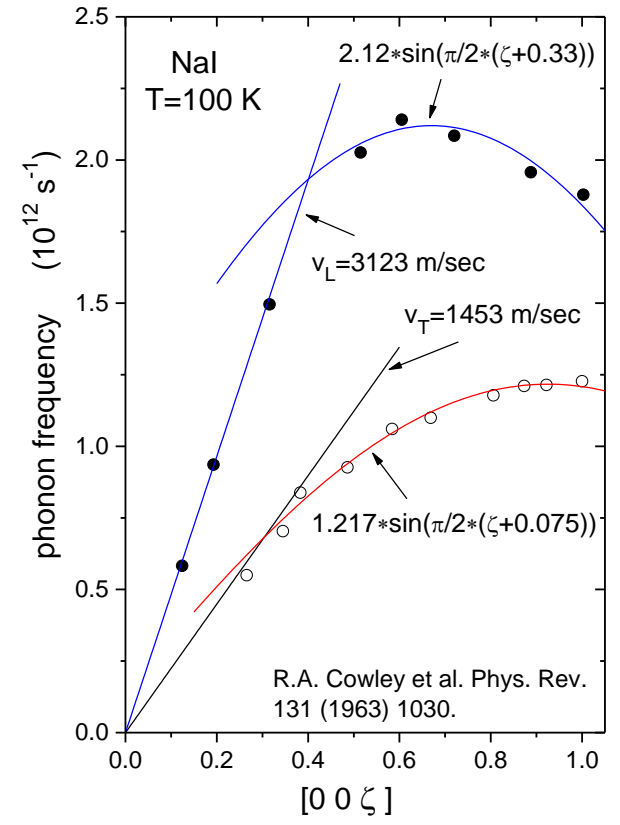

Figure 7. Dispersion of the acoustic phonons of NaI along cube edge for longitudinal and transverse polarization [20]. The linear dispersions agree with the measured sound velocities [7]. This shows that phonons are attracted by the dispersion of the Debye bosons. At the limits of the linear dispersion an analytical crossover to sine function of wave vector follows. To be consistent with the experimental data an absolute constant has to be added to the argument of the sine function.

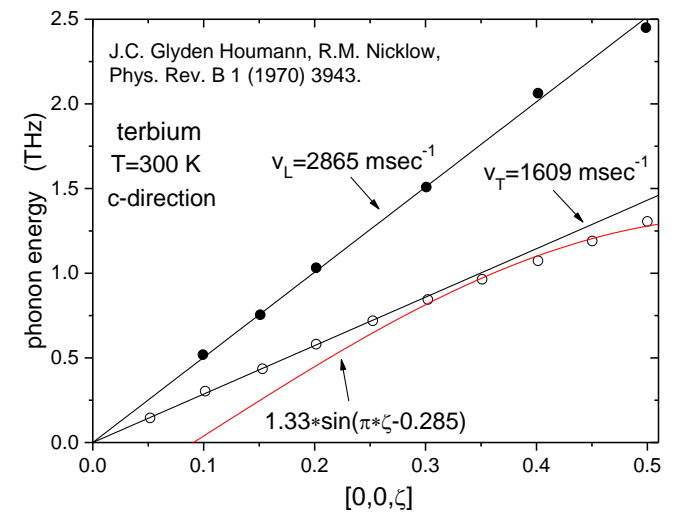

Figure 8. Acoustic phonon dispersions along hexagonal axis of terbium measured at $T=300 \mathrm{~K}\left(T_{c}=229 \mathrm{~K}\right)$ [21]. Within experimental accuracy the branch with longitudinal polarization has perfectly assumed the linear dispersion of the Debye bosons up to zone boundary. The slope of this line agrees with the measured sound velocity [7]. For transverse polarization linear dispersion holds for a smaller q-range only.

Another example of extremely strong Debye bosonphonon interactions is hexagonal terbium [21]. As Figure 8 shows, within experimental accuracy, the longitudinal acoustic phonon branch along hexagonal axis shows perfect linear wave vector dependence up to zone boundary. In other words, phonon dispersion has completely adapted to the nonpopulated Debye boson dispersion. The slope of this line agrees with the sound velocity, $v_{L}$ [7]. Data of Figure 8 are for $T=300 \mathrm{~K}$ which is above magnetic ordering temperature of terbium of $T_{c}=229 \mathrm{~K}$. For transverse polarization Debye boson-phonon interaction is weaker than for longitudinal polarization and the linear section of the phonon dispersion holds over a smaller wave vector range. Analytical crossover to sine function is not clearly resolved. Nevertheless fit by sine function including a phase shift gives reasonable description of the few experimental data points.

\section{Conclusions}

Solids have a twofold nature. On the one hand, we know from crystallography, that they are buildup of discrete atomistic units but, on the other hand, they exhibit properties as a continuous medium. The elastic properties of solids and thermal conductivity as well are typical macroscopic phenomena of the continuous or infinite solid. In the theory of elasticity atoms need not to be considered. Heat flow in solids can be described by a simple (macroscopic) differential equation. In other words, there are thermodynamic phenomena in solids, such as sound propagation or thermal conductivity, in which atoms and interactions between atoms are not involved. This is the proper definition of universality. Universality is the thermodynamic behavior of a field of freely propagating bosons and, so to say, a macroscopic phenomenon. In other words, elasticity and heat transport (in insulators) are due to Debye bosons. Note that thermal conductivity of insulators tends to zero for temperatures of larger than $10 \ldots 20 \mathrm{~K}$ where Debye bosons are no longer the relevant excitations.

The atomistic background is, however, not completely negligible. The absolute values of the sound velocities as well as the anisotropy of the elastic continuum are material specific characteristics due to the atomistic structure. Moreover, scattering at lattice defects provide a sample dependent damping to the Debye bosons and let sound velocity and heat transport depend on the structural perfection of the sample. Different sound velocities along different crystallographic directions could, formally, be described by an anisotropic index of refraction. The anisotropy of the elastic constants is material specific. In spite of anisotropic elastic constants (anisotropic sound velocities), the heat capacity of the Debye boson field always is $\sim T^{3}$.

As we have seen, the sources of the Debye bosons must have atomistic dimensions. This seems to apply to other types of field quanta as well. For instance, the sources of the electromagnetic radiation field are electric dipoles, commonly associated with transitions between different electronic orbitals. In magnetism the field quanta are magnetic dipole radiation emitted upon precession of the ordered spins [6]. In this communication we have obtained indication that the (spontaneous) sources of the Debye boson must be the individual atoms. Since the $T^{3}$ function of the heat capacity of the Debye boson field is observed also in strongly anisotropic crystals it can be concluded that the Debye boson field always is isotropic and therefore can be characterized as a scalar field. The radiation characteristic of the field sources therefore must essentially be isotropic. The sources of the Debye bosons must have spherical shape. It is tempting to conclude that the sources of the Debye bosons are the nearly spherical atomic cores [16,17]. Modeling the atomic core by a homogeneous elastic sphere, comparison with available calculations of the vibrational modes of such small spheres (quantum dots) is useful [22]. It is found that 
in small spheres there are two types of vibrational modes: a spheroidal or dilatation mode and a torsional or surface mode. The two modes are Raman active [23]. However, emission of sound waves by such vibrational modes is a completely unexplored process. Emission of field quanta requests coupling of the localized vibrational modes to the elastic continuum. On the other hand, it is clear that in addition to the electrostatic forces, gravitational forces are also important in atoms. Note that the radiation characteristic of the sources of the electromagnetic radiation field, the electric dipoles, is planar. In magnetism the radiation characteristic of the field sources, the processing magnetic moments, is axial. Magnetic dipole radiation is emitted along precession axis. This provides vector character to the field. The global boson field in ordered magnets can assume any dimension [15].

Debye bosons (sound waves) can be induced out of thermal equilibrium at high temperatures where they do not contribute to heat capacity. Equivalently, solids exhibit elastic properties at all temperatures rather independent of whether the Debye dispersion relation is thermally populated or not. Note that the elastic properties are reactions upon external manipulations of the sample. For temperatures of larger than $10 \ldots 20 \mathrm{~K}$ the Debye boson field is thermodynamically no longer relevant and its heat capacity tends to zero. Break down of the heat capacity of the boson field can be assumed to happen at crossover to phonons. Nevertheless the not populated dispersion of the Debye bosons can attract the dispersion of the acoustic phonons. This allows verification of the very nearly linear dispersion of the Debye bosons from inelastic neutron scattering data. Note that inelastic neutron scattering is incapable of detecting the mass less Debye bosons. For sound velocity and elastic constants as well it seems to be unimportant whether the dispersion of the Debye bosons is thermally populated or not.

The sound velocities that can be measured up to melting temperature [7] shows that the dispersion of the Debye bosons continues up to thermal energy of $k_{B} T_{m}$. This is a much larger energy than the largest phonon energy, characterized by Debye temperature $\Theta_{\mathrm{D}}$. Since the zone boundary is of no importance on the freely propagating bosons, the dispersion of the Debye bosons can continue beyond zone boundary. For LiF sound velocities at melting temperature of $T_{m}=1121 \mathrm{~K}$ are $v_{T}=4192 \mathrm{~m} / \mathrm{sec}$ and $v_{L}=3328$ $\mathrm{m} / \mathrm{sec}$ along [1,0,0] direction [7]. At $T_{m}$ the wavelengths of the sound waves are $\lambda_{\mathrm{T}} \sim 1.8 \AA$ and $\lambda_{\mathrm{L}} \sim 1.4 \AA$, respectively. This is less than half of the lattice parameter of $a_{0}=4.0173$ $\AA$. For metallic niobium with $T_{m}=2760 \mathrm{~K}$ the wavelengths of sound waves at melting temperature along $[1,0,0]$ direction are $0.82 \AA$ and $0.33 \AA\left(a_{0}=3.30 \AA\right)$. As a conclusion, at melting temperature the wavelength of the (nonrelevant) sound waves is of the order of the atomic diameters or even lower.

\section{References}

[1] P. Debye, Zur Theorie der spezifischen Wärmen, Ann. Physik 39, 789-839, 1912

[2] U. Köbler, A. Hoser, Renormalization Group TheoryImpact on Experimental Magnetism, Springer, Berlin, 2010.

[3] M. Born, K. Huang, Dynamical Theory of Crystal Lattices, Clarendon Press, Oxford, 1956.
[4] K.G. Wilson, J. Kogut, The renormalization group and the $\varepsilon$ expansion, Physics Reports 12C 75-200, 1974.

[5] K.G. Wilson, The renormalization group: Critical phenomena and the Kondo problem, Reviews of Modern Phys. 47, 773-840, 1975.

[6] U. Köbler, Towards a Field Theory of Magnetism in: K. Pace (Ed.), Recent Developments in Magnetism Research (Nova Science Publishers, Hauppauge, New York, 2013) pp. 1-66.

[7] R.F.S. Hearmon in: K.-H. Hellwege and A.M. Hellwege (Eds.), Landolt-Börnstein vol. 18 Springer, Berlin, p. 1, 1984.

[8] Y. Endoh, G. Shirane, J. Skalyo, Jr., Lattice dynamics of solid neon at 6.5 and 23.7 K, Phys Rev. B 11, 1681-1688, 1975.

[9] Y. Fujii, N.A. Lurie, R. Pynn, G. Shirane, Inelastic neutron scattering from solid ${ }^{36} \mathrm{Ar}$, Phys. Rev. B 10, $3647-$ 3659, 1974.

[10] J. Skalyo, Jr., Y. Endoh, G. Shirane, Inelastic neutron scattering from solid krypton at $10 \mathrm{~K}$, Phys. Rev. B 9, 1797-1803, 1974.

[11] N.A. Lurie, G. Shirane, J. Skalyo, Jr., Phonon dispersion relations in xenon at $10 \mathrm{~K}$, Phys. Rev. B 9 5300-5306, 1974.

[12] A.A.Z. Ahmad, H.G. Smith, N. Wakabayashi, M.K. Wilkinson, Lattice dynamics of cesium chloride, Phys. Rev. B 6, 3956-3961, 1972.

[13] G. Dolling, H.G. Smith, R.M. Nicklow, P.R. Vijayaraghavan, M.K. Wilkinson, Lattice dynamics of lithium fluoride, Phys. Rev. 168, 970-979, 1968.

[14] C.V. Briscoe, C.F. Squire, Elastic constants of LiF from 4.2 K to $300 \mathrm{~K}$ by ultrasonic methods, Phys. Rev. 106, 1175-1177, 1957.

[15] U. Köbler, Bosons and magnons in ordered magnets, Acta Phys. Pol. A 127, 1694-1701, 2015.

[16] H. Witte, E. Wölfel, Electron distributions in $\mathrm{NaCl}, \mathrm{LiF}$, $\mathrm{CaF}_{2}$ and $\mathrm{Al}$, Rev. Mod. Phys. 30, 51-55, 1958.

[17] G. Schoknecht, Röntgen-kristallstrukturanalyse mit faltungsintegralen, ii. meßverfahren und bestimmung der elektronendichte in $\mathrm{NaCl}, \mathrm{Z}$. Naturforschung 12a, 983996, 1957.

[18] B.M. Powell, P. Martel, A.D.B. Woods, Lattice dynamics of niobium-molybdenum alloys, Phys. Rev. 171, 727, 1968.

[19] K.A. Jones, S.C. Moss, R.M. Rose, The Effect of small oxygen additions on the elastic constants and low temperature ultrasonic attenuation of $\mathrm{Nb}$ single crystals, Acta Met. 17, 365-372, 1969.

[20] R.A. Cowley, W. Cochrane, B.N. Brockhouse, A.D.B. Woods, Lattice dynamics of alkali halide crystals. III. Theoretical, Phys. Rev. 131, 1030-1039, 1963.

[21] J.C. Glyden Houmann, R.M. Nicklow, Lattice Dynamics of Terbium, Phys. Rev. B 1, 3943-3952, 1970.

[22] T. Takagahara, "Electron-Phonon interactions in semiconductor quantum dots" in Semiconductor Quantum Dots, Y. Masumoto, T. Takagahara (Eds.) Berlin: Springer, pp. 115-147.

[23] A. Tanaka, S. Onari, T. Arai, Phys. Rev. B 47, $1237-$ $1243,1933$. 